\title{
CONSUMO ALIMENTAR E ESTADO NUTRICIONAL DE CRIANÇAS EM UMA ESCOLA PRIVADA DE PALMAS, TOCANTINS
}

Food consumption and nutritional status of a private school children at Palmas, Tocantins

Ingesta de alimentos y nutrición de los niños de una escuela privada de Palmas, Tocantins

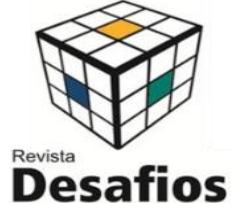

Artigo Original Original Article Artículo Original

Laynie Evangelina do Nascimento Menêses ${ }^{1}$, Nainny Vieira Silva ${ }^{1}$, Marina Rabelo Labre ${ }^{1}$, José Gerley Diaz Castro ${ }^{1}$, Juliano Vidal Barbosa Filho ${ }^{2}$, Renata Junqueira Pereira ${ }^{1^{*}}$

1 Curso de Nutrição, Universidade Federal do Tocantins, Palmas, Tocantins, Brasil.

2 Mestrado em Ciências da Saúde, da Universidade Federal do Tocantins, Palmas, Tocantins, Brasil.

* Correspondência: Laboratório de Nutrição, Universidade Federal do Tocantins, Av. NS 15, 109 Norte, Palmas, Tocantins, Brasil.CEP:77.010-090.e-mail: renatajunqueira@uft.edu.br

\section{RESUMO}

A identificação do perfil de consumo alimentar, bem como a do estado nutricional em escolares se faz muito importante, pois é nessa fase da vida que se formam os hábitos alimentares saudáveis, os quais contribuem para uma vida saudável até a fase adulta. Esse estudo teve como objetivo avaliar os hábitos de frequência do consumo alimentar e o perfil antropométrico de crianças, de 5 a 10 anos incompletos, de uma escola privada de Palmas, Tocantins. Foram avaliadas 184 crianças, de ambos os sexos, as quais foram submetidas a avaliação antropométrica e responderam a um questionário de frequência do consumo alimentar. Dos escolares avaliados, a maioria estava eutrófica, $37 \%$ apresentaram risco nutricional e 4,3\%, obesidade grave. O consumo alimentar mostrou-se semelhante entre os dois sexos e para todas as classes de estado nutricional. Os resultados ressaltaram a necessidade de educação alimentar e nutricional para essas crianças, incluindo os ambientes familiares e escolar.

Palavras-chave: Escolares. Consumo de Alimentos. Estado Nutricional.

\section{ABSTRACT}

The identification of the food consumption profile as well as the nutritional status in schoolchildren becomes very important, because it is during this phase of life that healthy eating habits are formed, which contribute to a healthy life until adulthood. The objective of this study was to evaluate the eating habits frequency and the anthropometric profile of children from 5 to 10 years of age, from a private school in Palmas, Tocantins. A total of 184 children, both boys and girls, who underwent an anthropometric evaluation and answered a food consumption form were evaluated. Of the students evaluated, most were eutrophic, 37\% presented nutritional risk and 4.3\%, severe obesity. Dietary intake was similar between both sexes and for all classes of nutritional status. The results highlighted the need for food and nutrition education for these children, including family and school settings.

Keywords: Schoolchildren. Food consumption. Nutritional Status.

\section{RESUMEN}

Identificación de perfil de consumo de alimentos, así como el estado nutricional de la escuela llega a ser muy importante porque es en esta etapa de la vida que se forman los hábitos alimenticios saludables, que contribuyen a una vida saludable a la edad adulta. Este estudio tuvo como objetivo evaluar la frecuencia de los hábitos de consumo de alimentos y el perfil antropométrico de niños de 5 a 10 años de edad, una escuela privada Palmas, Tocantins. Se evaluaron 184 niños, de ambos sexos, que fueron sometidos a una evaluación antropométrica y se les aplicó un tipo de consumo de alimentos. De los escolares, la mayoría eran eutróficos, 37\% tenían riesgo 
nutricional y el 4,3\%, obesidad severa. La ingesta de alimento fue similar entre los sexos y de todas las clases del estado nutricional. Los resultados pusieron de relieve la necesidad de una educación alimentaria y nutricional de estos niños, incluyendo los ambientes familiares y escolares.

Descriptores: Escuela. El consumo de alimentos. El estado nutricional.

\section{INTRODUÇÃO}

A alimentação adequada, em quantidade e qualidade, fornece ao organismo energia e nutrientes necessários para a manutenção de um bom estado de saúde. O consumo alimentar insuficiente é fator de risco para a desnutrição e deficiências de micronutrientes. Por outro lado, o excesso alimentar relaciona-se à ocorrência de obesidade e suas comorbidades, como hipertensão arterial, diabetes mellitus, dislipidemias e doenças cardiovasculares (BRASIL, 2016).

Segundo Cavalcante et al. (2004), a avaliação do consumo alimentar tem grande relevância nas áreas de nutrição e saúde. Entretanto, para avaliar o consumo alimentar são necessários métodos apropriados para se estimar a ingestão de alimentos e nutrientes de grupos populacionais (MESSIAS, SOUZA E REIS, 2016).

Em relação às crianças e adolescentes, se faz especialmente importante avaliar seu consumo alimentar, pois tais etapas do ciclo vital caracterizamse por diversas alterações de crescimento e composição corporal (RIVERA; SOUZA, 2006). Na infância, além da criança exercer pouco controle sobre a disponibilidade domiciliar de alimentos, pode sofrer influências dos hábitos alimentares e de atividade física dos pais e familiares. Além disso, está propensa a alterações de comportamento alimentar, devido à sua inserção no ambiente escolar (UNIVERSITY OF SIDNEY, 2005; FERNANDES et al., 2009).

A identificação do perfil de consumo alimentar, bem como do estado nutricional em escolares, constitui, portanto, uma etapa fundamental para o estabelecimento de estratégias, com o objetivo de reverter o atual quadro epidemiológico nutricional, visando a promoção da saúde e da alimentação saudável (FALCÃO-GOMES; COELHO; SCHMITZ, 2006).

Considerando-se que a literatura referente ao estudo do consumo alimentar de crianças brasileiras é escassa, e sabendo que no Brasil tem sido detectada a progressão da transição nutricional em escolares, o objetivo do presente trabalho foi avaliar os hábitos de frequência do consumo alimentar e $\mathrm{o}$ perfil antropométrico de crianças, de 5 a 10 anos incompletos, de uma escola privada de Palmas, Tocantins.

\section{MATERIAL E MÉTODOS}

O trabalho foi desenvolvido por meio da coleta de dados antropométricos e aplicação de um formulário de marcadores do consumo alimentar, em 184 crianças, de 5 a 10 anos incompletos, matriculadas em uma escola privada de Palmas-TO. A faixa etária selecionada para o estudo foi baseada na estratificação proposta em Brasil (2008).

Inicialmente, as crianças foram abordadas pela equipe pedagógica da escola, afim de se esclarecerem os procedimentos para as tomadas de peso e altura e a necessidade de resposta ao questionário. Tal ação foi realizada por meio de exposição teórica e audiovisual (dois vídeos) (INADPCRJ, 2012a; INADPCRJ, 2012b). Aqueles alunos que aceitaram participar do estudo assinaram o termo de assentimento e levaram para casa o termo de consentimento livre e esclarecido, endereçado aos pais e/ou responsáveis, para que os mesmos permitissem que seus filhos participassem da pesquisa. $O$ protocolo desse estudo foi aprovado pelo Comitê de Ética em 
Pesquisa da Universidade Federal do Tocantins, sob o número 075/2014.

Os escolares tiveram sua antropometria avaliada pela obtenção de peso e estatura. A pesagem foi realizada com as crianças colocadas no centro da balança, descalças, eretas, com os pés juntos e os braços estendidos ao longo do corpo. Já para a mensuração da estatura, as crianças foram colocadas descalças, em posição ereta, encostadas em uma superfície plana vertical, com os braços pendentes e com as mãos espalmadas sobre as coxas, os calcanhares unidos e as pontas dos pés afastadas, formando ângulo de $60^{\circ}$; joelhos em contato, cabeça ajustada ao plano de Frankfurt e em inspiração profunda. A medida foi tomada utilizando-se o estadiômetro, fixado à parede sem rodapé.

Os equipamentos utilizados para aferição de peso e altura foram cedidos pela Universidade Federal do Tocantins, Campus de Palmas e se trataram de uma balança digital (Marca: Tech Line - BAL - 10), com capacidade máxima de $180 \mathrm{Kg}$ e precisão de $100 \mathrm{~g}$; e um estadiômetro portátil, de alta resistência (Marca Wiso), com campo de medição de 0 a $210 \mathrm{~cm}$ de altura.

A avaliação do estado nutricional foi realizada por meio do uso do software WHO Anthro Plus (versão 3.2.2), nas curvas de Índice de Massa Corporal para a Idade (IMC/Idade) (WHO, 2007).

Além da antropometria, foram obtidas também respostas ao questionário de frequência do consumo alimentar, proposto pelo Sistema de Vigilância Alimentar e Nutricional - SISVAN, para avaliação desse grupo etário (BRASIL, 2008). A escolha do questionário de frequência de consumo alimentar a ser utilizado, levou em consideração a recomendação de uso pelo SISVAN para crianças dessa faixa etária.
O referido questionário apresenta algumas opções de alimentos e bebidas, relacionados tanto a uma alimentação saudável (exemplo: consumo diário de feijão, frutas, verduras) como às práticas pouco recomendadas (exemplo: consumo frequente de alimentos fritos e guloseimas). Esses alimentos foram apresentados às crianças por meio de gravuras que os pesquisadores utilizaram ao entrevistarem individualmente cada criança para obtenção das respostas de frequência alimentar.

Após a coleta, os dados foram tabulados e os grupos de alimentos foram numerados de 1 a 10 (conforme apresentados no formulário utilizado), para facilitar a leitura:

1. Salada crua;

2. Legumes e verduras cozidos;

3. Frutas frescas ou salada de frutas;

4. Feijão;

5. Leite ou iogurte;

6. Batata frita, batata de pacote e salgadinhos fritos;

7. Hambúrguer e embutidos;

8. Bolachas/biscoitos salgados ou salgadinhos de pacote;

9. Bolachas/biscoitos doces ou recheados, doces, balas e chocolates;

10. Refrigerante (desconsiderando os diets ou light).

O consumo alimentar foi classificado, conforme a frequência com que tais alimentos foram consumidos nos últimos sete dias, em: não consumiram, consumo esporádico (entre 1 e 4 dias) e consumo frequente (por 5 dias ou mais).

Os dados foram organizados para análise estatística descritiva, sendo agrupados conforme sexo dos escolares, frequência do consumo alimentar e classificação do estado nutricional. 


\section{RESULTADOS E DISCUSSÃO}

Das 184 crianças avaliadas, 87 eram do sexo mostrou que o estado nutricional que prevaleceu foi o de eutrofia, seguido do sobrepeso, em ambos os sexos, tal como mostra a Tabela 1.

feminino e 97 do sexo masculino. No que se refere aos dados antropométricos, o índice IMC/Idade

Tabela 1. Percentuais de classificação do estado nutricional das crianças, conforme IMC/idade.

\begin{tabular}{lcc}
\hline Estado Nutricional & Meninas & Meninos \\
\hline Eutrofia & $63 \%$ & $63 \%$ \\
Sobrepeso & $26 \%$ & $24 \%$ \\
Obesidade & $7 \%$ & $8 \%$ \\
Obesidade grave & $3 \%$ & $5 \%$ \\
Total & $100 \%$ & $100 \%$ \\
\hline
\end{tabular}

*Nenhuma criança apresentou magreza acentuada ou magreza para o critério utilizado.

Tabela 2. Percentuais de consumo dos grupos de alimentos de ambos os sexos

\begin{tabular}{ccccccc}
\hline & \multicolumn{3}{c}{ Meninas } & \multicolumn{3}{c}{ Meninos } \\
\hline $\begin{array}{c}\text { Grupos de } \\
\text { Alimentos }\end{array}$ & $\begin{array}{c}\text { Não } \\
\text { consumiram }\end{array}$ & $\begin{array}{c}\text { Consumo } \\
\text { esporádico }\end{array}$ & $\begin{array}{c}\text { Consumo } \\
\text { frequente }\end{array}$ & $\begin{array}{c}\text { Não } \\
\text { consumiram }\end{array}$ & $\begin{array}{c}\text { Consumo } \\
\text { esporádico }\end{array}$ & $\begin{array}{c}\text { Consumo } \\
\text { frequente }\end{array}$ \\
$\mathbf{1}$ & $9,20 \%$ & $35,63 \%$ & $55,17 \%$ & $21,65 \%$ & $41,24 \%$ & $37,11 \%$ \\
$\mathbf{2}$ & $27,59 \%$ & $48,28 \%$ & $24,14 \%$ & $34,02 \%$ & $45,36 \%$ & $20,62 \%$ \\
$\mathbf{3}$ & $3,45 \%$ & $28,74 \%$ & $67,82 \%$ & $4 \%$ & $40,21 \%$ & $55,67 \%$ \\
$\mathbf{4}$ & $6,90 \%$ & $9,20 \%$ & $83,91 \%$ & $5,15 \%$ & $10,31 \%$ & $84,54 \%$ \\
$\mathbf{5}$ & $2,30 \%$ & $21,84 \%$ & $75,86 \%$ & $6,19 \%$ & $13,40 \%$ & $80,41 \%$ \\
$\mathbf{6}$ & $13,79 \%$ & $74,71 \%$ & $11,49 \%$ & $14,43 \%$ & $77,32 \%$ & $8,25 \%$ \\
$\mathbf{7}$ & $20,69 \%$ & $68,97 \%$ & $10,34 \%$ & $29,90 \%$ & $61,86 \%$ & $8,25 \%$ \\
$\mathbf{8}$ & $19,54 \%$ & $59,77 \%$ & $20,69 \%$ & $17,53 \%$ & $59,79 \%$ & $8,25 \%$ \\
$\mathbf{9}$ & $14,94 \%$ & $59,77 \%$ & $25,29 \%$ & $15,46 \%$ & $68,04 \%$ & $16,49 \%$ \\
$\mathbf{1 0}$ & $20,69 \%$ & $64,37 \%$ & $14,94 \%$ & $23,71 \%$ & $57,73 \%$ & $18,56 \%$ \\
\hline
\end{tabular}

Em relação à frequência de consumo dos alimentos, os mais consumidos foram os do grupo 4 (Feijão), seguidos daqueles dos grupos 5 (Leite ou iogurte) e 3 (Frutas frescas ou salada de frutas), para ambos os sexos. No consumo esporádico, houve predominância dos alimentos do grupo 6 (Batata frita, 
batata de pacote e salgadinhos fritos), para os dois sexos; seguidos dos grupos 7 (Hambúrguer e embutidos) e 8 (Refrigerantes) entre as meninas, e do grupo 9 (Bolachas/biscoitos doces ou recheados, doces, balas e chocolates), entre os meninos; enquanto na classificação "não consumiram", os alimentos que prevaleceram foram os do grupo 2 (Legumes e verduras cozidas), para ambos os sexos, como mostrado na Tabela 2.

Os Gráficos 1 e 2 mostram que, tanto no grupo feminino, quanto no masculino, ambos eutróficos, os alimentos que foram consumidos com maior frequência foram os dos grupos 4 (Feijão) e 5 (Leite ou iogurte). Em contrapartida, houve uma grande prevalência de consumo esporádico dos alimentos do grupo 6 (Batata frita, batata de pacote e salgadinhos fritos), seguidos daqueles do grupo 7 (Hambúrguer e embutidos) entre as meninas, e daqueles do grupo 9 (Bolachas/biscoitos doces ou recheados, doces, balas e chocolates), entre os meninos.

Gráfico 1. Frequência de consumo alimentar de crianças do sexo feminino, classificadas como eutróficas.

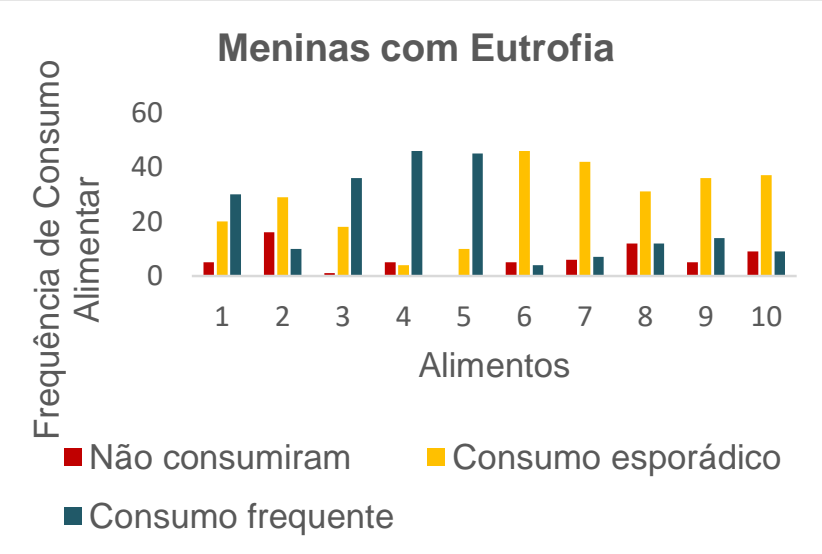

Gráfico 2. Frequência de consumo alimentar de crianças do sexo masculino, classificadas como eutróficas.

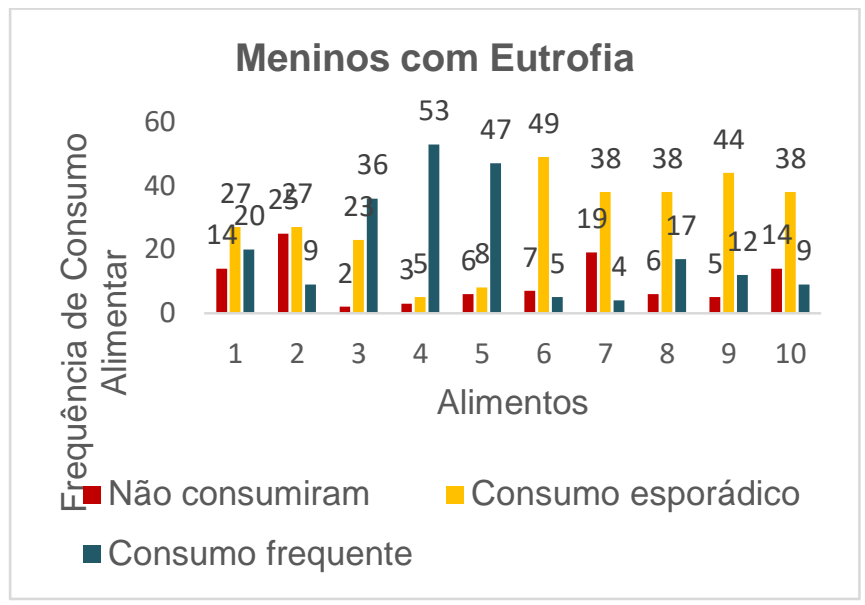

Os Gráficos 3 e 4, que dizem respeito às meninas e aos meninos com sobrepeso, respectivamente, mostram que, dentre as meninas, os alimentos mais frequentemente consumidos foram os dos grupos 4 (Feijão) e 3 (Frutas frescas ou salada de frutas) e, esporadicamente, os do grupo 8 (Bolachas/biscoitos salgados ou salgadinhos de pacote); enquanto dentre os meninos, os grupos 5 (Leite ou iogurte) e 4 (Feijão) tiveram maior consumo frequente, e os grupos 6 (Batata frita, batata de pacote e salgadinhos fritos) e 9 (Bolachas/biscoitos doces ou recheados, doces, balas e chocolates), se destacaram com maior consumo esporádico.

Gráfico 3. Frequência de consumo alimentar de crianças do sexo feminino classificadas com sobrepeso.

\section{Meninas com Sobrepeso}

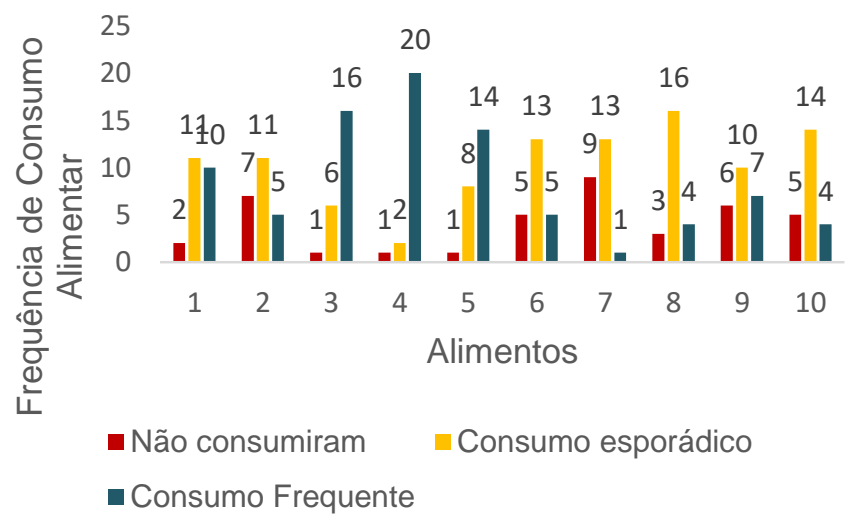


Gráfico 4. Frequência de consumo alimentar de crianças do sexo masculino classificadas com sobrepeso.

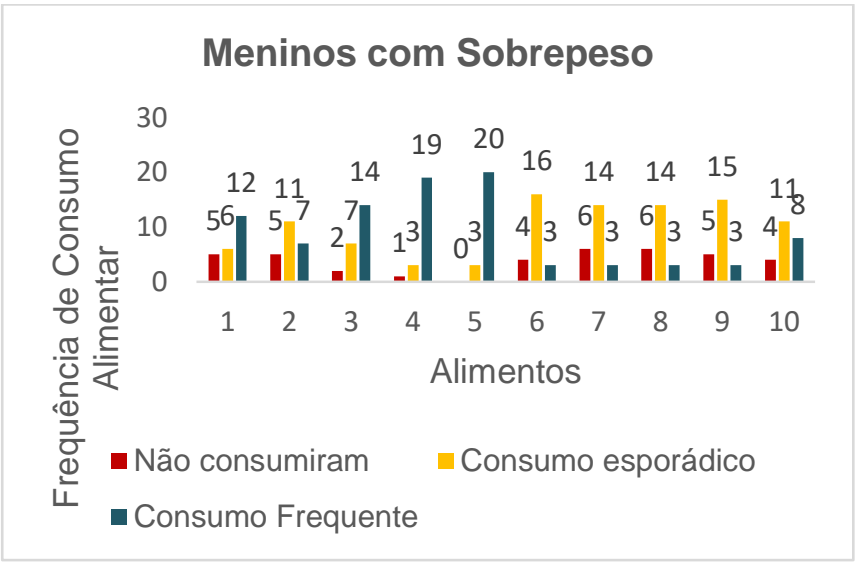

De acordo com os Gráficos 5 e 6, referentes às crianças com obesidade (ambos os sexos), os grupos 1 (Salada crua) e 3 (Frutas frescas ou salada de frutas) foram os grupos de alimentos consumidos com maior frequência entre as meninas, e o consumo esporádico foi maior do grupo 9 (Bolachas/biscoitos doces ou recheados, doces, balas e chocolates). Entre os meninos, o grupo de alimentos mais frequentemente consumido foi o 4 (Feijão), seguido do 5 (Leite ou iogurte). Os grupos de alimentos consumidos esporadicamente pelos meninos foi o 6 (Batata frita, batata de pacote e salgadinhos fritos), seguido do 7 (Hambúrguer e embutidos).

Gráfico 5. Frequência de consumo alimentar de crianças do sexo feminino classificadas com obesidade.

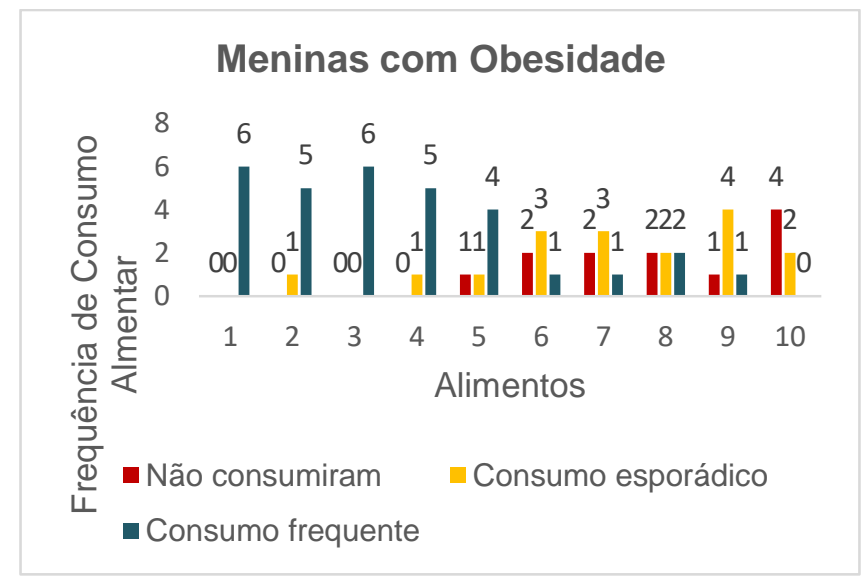

Gráfico 6. Frequência de consumo alimentar de crianças do sexo masculino classificadas com obesidade.

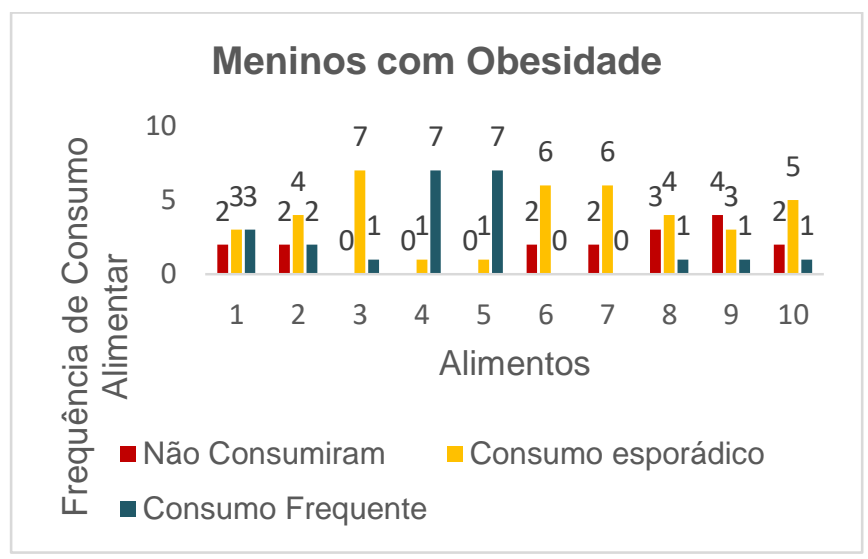

O grupo alimentar mais consumido entre as meninas e os meninos, classificados com obesidade grave, foi o 5 (Leite ou iogurtes), como mostram os Gráficos 7 e 8 . No entanto, as meninas consumiram, esporadicamente, mais alimentos dos grupos 6 (Batata frita, batata de pacote e salgadinhos fritos), 8 (Bolachas/biscoitos salgados ou salgadinhos de pacote) e 10 (Refrigerante), enquanto os meninos consumiram os do grupo 9 (Bolachas/biscoitos doces ou recheados, doces, balas e chocolates).

Gráfico 7. Frequência de consumo alimentar de crianças do sexo feminino classificadas com obesidade grave.

\section{Meninas com Obesidade Grave}

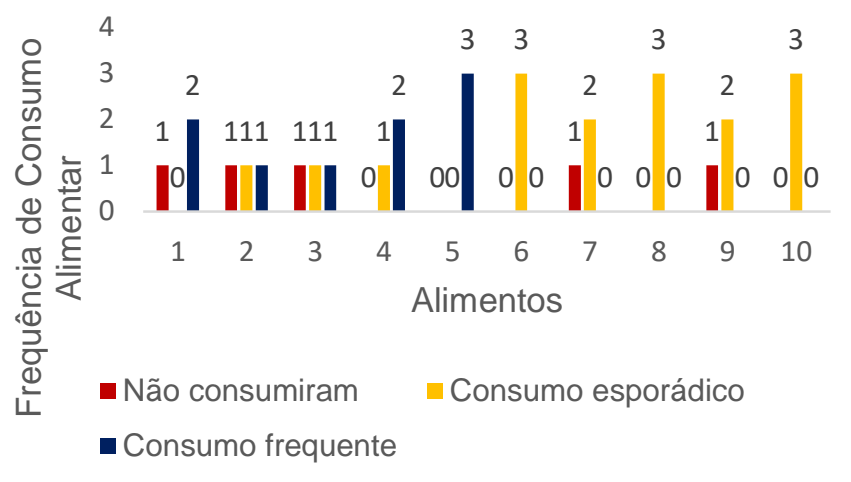


Gráfico 8 - Frequência de consumo alimentar de crianças do sexo masculino classificadas com obesidade grave.

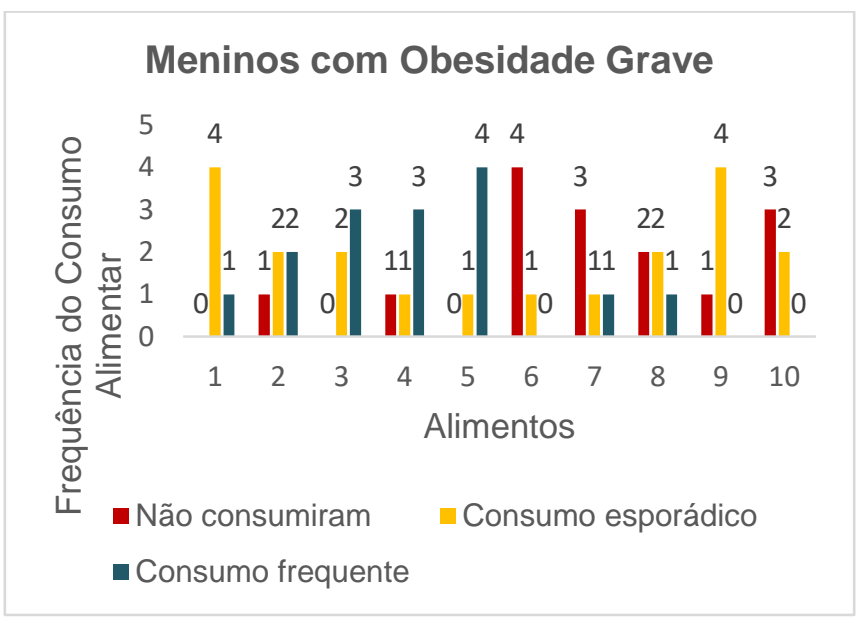

Das 184 crianças que participaram da pesquisa, 37\% apresentavam algum tipo de risco nutricional, sendo que 4,3\% apresentavam obesidade grave. Em relação ao sexo, não houve diferença entre os resultados do estado nutricional, porém a maior parcela dos riscos nutricionais (19\%) está entre as crianças do sexo masculino. Os dados dessa pesquisa confirmam uma tendência mundial, onde o número de pessoas obesas e com sobrepeso supera o de desnutridas. Nos últimos anos, a obesidade aumentou entre as crianças e segundo Kochi (2004) ela é decorrente de fatores genéticos, ambientais e psicossociais.

No tocante aos hábitos alimentares, notou-se que não houve diferença entre os sexos. Os alimentos mais consumidos, nos dois grupos, foram o feijão, o leite ou iogurte e as frutas frescas ou salada de frutas, enquanto os legumes e verduras cozidas tiveram menor prevalência de consumo.

Steil e Poll (2017), também estudando escolares, observaram 58,2\% de indivíduos eutróficos e $38,2 \%$ em excesso de peso. Verificaram também um consumo diário de carne, feijão e frutas por um maior número de escolares, já verduras, leite, guloseimas, refrigerante e sucos artificiais foram mais citados como consumidos "de vez em quando".
Houve também na população aqui estudada, um alto consumo esporádico de alimentos hipercalóricos e ricos em sódio, tais como bolachas/biscoitos doces ou recheados, doces, balas e chocolates, batata frita, batata de pacote, salgadinhos fritos, hambúrguer, embutidos e refrigerantes, sendo a maioria consumidos no horário do lanche, em substituição ao jantar e nos finais de semana.

Ressalta-se que o consumo de alimentos hipercalóricos e com altos teores de açúcar e sal estão associados ao aparecimento de doenças crônicas, tanto na própria infância como na vida adulta (BLOOM et al., 2015; BRASIL, 2016).

Em um estudo realizado por Conceição et al. (2010), concluiu-se que a proporção de consumo de biscoitos pelos escolares foi elevada $(52,6 \%)$. No presente estudo, o percentual de consumo dos biscoitos foi maior $(68,04 \%$ entre meninos e $59,77 \%$ entre meninas).

Ainda na pesquisa de Conceição et al. (2010), houve baixo consumo de embutidos (6,6\%). Em contrapartida, os embutidos apresentaram um elevado consumo esporádico nesse estudo (acima de 60\% nos dois grupos).

Da mesma forma, Messias, Souza e Reis (2016), estudando adolescentes, observaram que uma ingestão significativa de alimentos ricos em gorduras, açúcares, sódio e corantes de alimentos. Dentre os alimentos ricos em sódio mais citados foram os embutidos. Já os ricos em açúcares foram o achocolatado em pó e os biscoitos recheados.

Correlacionando o estado nutricional com o consumo alimentar, vemos que as crianças eutróficas, com sobrepeso, obesidade e obesidade grave consumiram com maior frequência os mesmos alimentos. Concordam com esses resultados os do estudo de Novaes, Franceschini e Priore (2007), que observaram que os hábitos alimentares foram semelhantes entre crianças eutróficas e aquelas com 
sobrepeso, ou seja, grande parte dos alimentos consumidos era similar entre os dois grupos.

Steil e Poll (2017) também verificaram frequências de consumo de guloseimas, sucos artificiais e refrigerantes semelhantes entre escolares eutróficos e com excesso de peso.

Como pode-se notar, o consumo alimentar dos escolares que participaram desse levantamento, apresentou tanto aspectos favoráveis, como negativos, o que revela a falta da educação alimentar e nutricional para essas crianças, bem como para os pais e a escola, uma vez que os hábitos alimentares são condicionados na infância pelos pais, escola e pelos meios de comunicação (VAZ; BENNEMANN, 2014).

\section{CONCLUSÃO}

É durante a infância que os hábitos alimentares saudáveis são formados e eles previnem o desenvolvimento de doenças crônicas não transmissíveis até a vida adulta. Nesse estudo, observou-se um elevado consumo esporádico de alimentos hipercalóricos, apesar de a maioria das crianças serem classificadas como eutróficas. Tal fato ressalta a importância da educação alimentar e nutricional a esse grupo, pois a ausência de medidas que promovam a melhora do comportamento alimentar, pode ser fator de risco para aumento dos casos de sobrepeso e obesidade.

Tal intervenção, no entanto, não deve envolver apenas as crianças, mas também as famílias e a escola, a fim de se obterem melhores resultados, já que esses grupos exercem enorme influência sobre os hábitos alimentares dos escolares.

Nesse sentido, a educação nutricional tornase essencial, posto que ela busca a modificação dos hábitos alimentares no longo prazo, e pode ser utilizada como um elemento de conscientização e reformulação das distorções do comportamento alimentar, auxiliando a refletir sobre a saúde e a qualidade de vida (MANTOANELLI et al., 1997; CANO et al., 2005).

Todos os autores declararam não haver qualquer potencial conflito de interesses referente a este artigo.

\section{REFERÊNCIAS}

BRASIL. Ministério da Saúde. Secretaria de Atenção à Saúde. Departamento de Atenção Básica. Protocolos do Sistema de Vigilância Alimentar e Nutricional - SISVAN na assistência à saúde. Brasília: Ministério da Saúde, 2008. 61p.

BRASIL. Ministério da Saúde. Secretaria de Atenção à Saúde. Departamento de Atenção Básica. Caderno de Atenção Básica $\mathbf{n}^{\mathbf{0}}$ 23. Brasília: Ministério da Saúde, 2016.

BLOOM, M. I. T; SANTOS, T. M. P.; ATAIDESILVA, T.; VASCONCELOS, S. M. L. Ingestão de vitaminas e minerais em uma amostra de hipertensos de um município da região nordeste do Brasil. Rev Bras Nutr Clin, v.30, n.2, p.154-8, 2015.

CONCEIÇÃO, S. I. O.; SANTOS, C. J. N.; SILVA, A. A. M.; SIlVA, J. S.; OllVEIRA, T. C. Consumoalimentar de escolares das redes pública e privada de ensino de São Luís, Maranhão. Revista de Nutrição, v. 23, n. 6, p. 993-1004, nov./dez. 2010.

FALCÃO-GOMES, R. C.; COELHO, A. A. S.; SCHMITZ, B. de A. S. Caracterização dos estudos de avaliação do consumo alimentar de pré-escolares. Revista de Nutrição, v. 19, n. 6, p. 713-727, nov./dez. 2006.

FERNANDES, P. S.; BERNARDO, C. O.; CAMPOS, R. M. M. B.; VASCONCELOS, F. A. G. Avaliação do efeito da educação nutricional na prevalência de sobrepeso/obesidade e no consumo alimentar de escolares do ensino fundamental. Jornal de Pediatria, v. 85, n. 4, p. 315-321, maio 2009.

KOCHI, C. Escola saudável dá certo. Revista CRN-

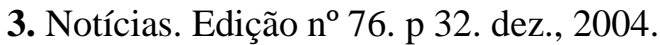

Medindo crianças maiores de 2 anos. INADPCRJ, 2012a. 1 vídeo eletrônico (1 min. e 11 seg.): son., color. Port. Disponível em: <http://www.youtube.com/watch?v=3_dKMAyyALY >. Acesso em: 13 de agosto de 2014. 
MESSIAS, C. M. B. O.; SOUZA, H. M. S.; REIS, I. R. M. S. Consumo de alimentos ultraprocessados e corantes alimentares por adolescentes de uma escola pública. Revista Adolescência \& Saúde, v. 13, n. 4, p. 7-14, 2016.

NOVAES, J. F.; FRANCESCHINI, S. C. C.; PRIORE, S. E. Hábitos alimentares de crianças eutróficas e com sobrepeso em Viçosa, Minas Gerais, Brasil. Revista de Nutrição, v.20, n. 6, p. 633-642, nov/dez, 2007.

Pesando crianças maiores de 2 anos. INADPCRJ, 2012b. 1 vídeo eletrônico (1 min. e 26 seg.): son., color. Port. Disponível em: < https://www.youtube.com/watch?v=ZqYBXrUmLKk >. Acesso em: 13 de agosto de 2014.

RIVERA, F. S. R.; SOUZA, E. M. T. Consumo alimentar de escolares de uma comunidade rural. Comum. Ciênc. Saúde, v.17, n. 2, p. 111-119, 2006.
STEIL, W. F.; POLL, F. A. Estado nutricional, práticas e conhecimentos alimentares de escolares. Cinergis, v.18, n.3, p.226-232, 2017.

UNIVERSITY OF SIDNEY. NEW SOUTH WALES CENTRE FOR PUBLIC HEALTH NUTRITION. Best options for promoting healthy weight and preventing weight gain in NSW. New South Wales: University of Sidney; 2005. 104p.

VAZ, D. S. S.; BENNEMANN, R. M.

Comportamento alimentar e hábito alimentar: uma revisão. Revista Uningá, v.20, n.1, p.108-112, 2014.

WORLD HEALTH ORGANIZATION. The WHO Reference 2007. Growth reference data for 5-19 years. Genova: WHO, 2007. Disponível em: <http://www.who.int/growhtref/en/>. Acesso em: 13 de setembro de 2014. 